\title{
Infectivity of severe acute respiratory syndrome coronavirus 2 in children compared with adults
}

\author{
Jared Bullard MD, Duane Funk MD, Kerry Dust PhD, Lauren Garnett BSc, Kaylie Tran BSc, Alex Bello PhD, \\ James E. Strong MD PhD, Santina J. Lee MD, Jillian Waruk PhD, Adam Hedley BSc, David Alexander PhD, \\ Paul Van Caeseele MD, Carla Loeppky PhD, Guillaume Poliquin MD PhD
}

Cite as: CMAJ 2021 April 26;193:E601-6. doi: 10.1503/cmaj.210263; early-released April 9, 2021

\begin{abstract}
BACKGROUND: The role of children in the transmission and community spread of severe acute respiratory syndrome coronavirus 2 (SARS-CoV-2) is unclear. We aimed to quantify the infectivity of SARSCoV-2 in nasopharyngeal samples from children compared with adults.
\end{abstract}

METHODS: We obtained nasopharyngeal swabs from adult and pediatric cases of coronavirus disease 2019 (COVID-19) and from their contacts who tested positive for SARS-CoV-2 in Manitoba between March and December 2020. We compared viral growth in cell culture, cycle threshold values from the reverse transcription polymerase chain reaction (RT-PCR) of the SARS-CoV-2 envelope (E) gene and the $50 \%$ tissue culture infective dose $\left(\mathrm{TCID}_{50} / \mathrm{mL}\right)$ between adults and children.

RESULTS: Among 305 samples positive for SARS-CoV-2 by RT-PCR, 97 samples were from children aged 10 years or younger, 78 were from children aged 11-17 years and 130 were from adults ( $\geq 18 \mathrm{yr}$ ). Viral growth in culture was present in $31 \%$ of samples, including 18 (19\%) samples from children 10 years or younger, 18 (23\%) from children aged 11-17 years and 57 (44\%) from adults (children v. adults, odds ratio 0.45 , 95\% confidence interval $[\mathrm{Cl}] 0.28-0.72)$. The cycle threshold was 25.1 (95\% Cl 17.731.3 ) in children 10 years or younger, 22.2 (95\% Cl 18.3-29.0) in children aged 11-17 years and 18.7 (95\% Cl 17.9-30.4) in adults $(p<0.001)$. The median $\mathrm{TCID}_{50} / \mathrm{mL}$ was significantly lower in children aged 11-17 years (316, interquartile range [IQR] 178-2125) than adults (5620, IQR 1171 to $17800, p<0.001)$. Cycle threshold was an accurate predictor of positive culture in both children and adults (area under the receiver-operator curve, $0.87,95 \% \mathrm{Cl} 0.81-0.93$ v. $0.89,95 \% \mathrm{Cl}$ $0.83-0.96, p=0.6)$.

INTERPRETATION: Compared with adults, children with nasopharyngeal swabs that tested positive for SARSCoV-2 were less likely to grow virus in culture, and had higher cycle thresholds and lower viral concentrations, suggesting that children are not the main drivers of SARS-CoV-2 transmission. evere acute respiratory syndrome coronavirus 2 (SARSCoV-2) and the nonpharmaceutical public health interventions (NPIs) to control it have had a considerable impact on society. Public health efforts directed to reduce the spread of coronavirus disease 2019 (COVID-19) have employed a number of NPIs, including suspension of in-person school attendance for school-aged children. These decisions were largely based on historical observations that children played a substantial role as drivers of transmission for epidemic respiratory viruses, such as influenza. ${ }^{1}$ In the case of SARS-CoV-2, the role of children in transmission remains unclear, given few studies with conflicting data. ${ }^{2-9}$ Most studies have been limited to epidemiological investigations from which the direction of transmission is challenging to discern. ${ }^{3-7,9}$ As an alternative line of evidence, some studies have investigated the role of SARSCoV-2 viral dynamics, also with heterogeneous results. Of these studies, some have shown higher viral loads in the nasopharynx of pediatric cohorts based on polymerase chain reaction testing, with others showing comparable levels of SARS-CoV-2 in children and adults. ${ }^{2,8,10,11}$ Furthermore, evidence relating to other viruses has shown that detectable viral RNA can persist beyond infectivity. ${ }^{3,4}$ An important proxy of in vivo infectiousness is recovery of live virus on cell culture. Assessment of this critical dimension has been lacking in virtually all pediatric 
studies, limiting the ability to perform a more complete riskbenefit analysis when considering the role of children in SARSCoV-2 transmission. Evidence shows that the infectivity of SARS-CoV-2 may be predicted using available data, such as the cycle threshold from the reverse transcription polymerase chain reaction (RT-PCR). ${ }^{12,13}$ Cycle threshold is a relative measure of the quantity of genetic material, with lower values indicating the presence of more viral genetic material in the sample.

As an increasing number of jurisdictions consider whether inschool learning, daycares and extracurricular activities should continue or resume, a better understanding of the relative contributions of children and adolescents to SARS-CoV-2 transmission, when compared with adults, is essential. This is particularly important given the increased likelihood of asymptomatic infection in this group. ${ }^{14,15}$ Our goal was to quantify rates of SARSCoV-2 culture positivity from nasopharyngeal swabs positive for the virus after RT-PCR testing in children. We then characterized the viral load and titres in culture-positive specimens and compared this with an adult group.

\section{Methods}

\section{Study population and design}

Beginning in July 2020, the province of Manitoba, Canada (population 1.4 million) had large-scale outbreaks of COVID-19. In efforts to limit transmission, comprehensive testing of case contacts was performed. Definitions of COVID-19 cases and case contacts are provided in Appendix 1, available at www.cmaj.ca/ lookup/doi/10.1503/cmaj.210263/tab-related-content.

We obtained nasopharyngeal swabs from patients with COVID-19 and their contacts. Sample RT-PCR testing was performed by the Cadham Provincial Laboratory, the reference laboratory for SARS-CoV-2 testing in Manitoba. Specimens were collected at COVID-19 testing sites and transported in viral transport medium to the laboratory, typically $1-4$ days after collection. In the laboratory, the specimens were stored at $4^{\circ} \mathrm{C}$ for 24 hours until they were tested as previously described. ${ }^{12}$ All samples were tested using laboratory-developed testing to minimize cycle threshold variation.

We submitted all specimens from children positive for SARSCoV-2 after RT-PCR for cell culture from March to August 2020. As case numbers increased, a convenience sample of positive specimens was provided to the National Microbiology Laboratory for cell culture analysis. We selected specimens from the preceding week's samples to ensure freshness and thereby maximize yields from cell culture. In November, we purposely selected pediatric specimens with a cycle threshold of less than 25 to confirm our preliminary observation that culture positivity rates were lower than adult samples (all pediatric sampling from Mar. 27 to Nov. 8, 2020). Cycle threshold values less than 25 were previously determined to have higher culture yields. ${ }^{12}$ Concurrently, we selected a convenience sample of adult specimens with cycle threshold values of 25 or less for comparison. Before the final cell culture analysis, we selected a convenience sample of specimens from adults (collected Mar. 12 to Dec. 14, 2020) for cell culture from the same health regions as pediatric samples.

\section{Outcomes}

Our main outcomes were culture positivity rates, RT-PCR cycle theshold values, the $50 \%$ tissue culture infectious dose $\left(\mathrm{TCI}_{50} / \mathrm{mL}\right)$, viral load (log RNA copies/mL) and symptoms to test time. For all positive samples, we obtained the RT-PCR cycle threshold values of the SARSCoV-2 envelope (E) gene. We also obtained the cycle threshold values of the human RNAse $\mathrm{P}$ gene, an endogenous internal amplification control used as a marker of quality of the nasopharyngeal sample.

The $\mathrm{TCID}_{50} / \mathrm{mL}$ assay is one method for quantifying infectious virus titres. Specifically, it quantifies the amount of virus required to kill $50 \%$ of tissue culture cells, thereby producing a cytopathic effect. Most samples were stored at $-80^{\circ} \mathrm{C}$ for 2 weeks before being processed for culture. Viral titres of samples were determined by the National Microbiology Laboratory (biocontainment level 4) using $\mathrm{TCID}_{50} / \mathrm{mL}$ assays (full methodology described in Appendix 1). In brief, serially diluted samples were placed on Vero cells and incubated for $96-120$ hours at $37^{\circ} \mathrm{C}$ and in $5 \% \mathrm{CO}_{2}$ before the $T C I D_{50}$ was measured.

Viral load is commonly measured as the logarithmic number of RNA genome copies per millilitre (log RNA copies $/ \mathrm{mL}$ ), a more standardized quantitative value than cycle thresholds. For this study, and to quantify the amount of viral RNA present in each sample, we generated a standard curve using a known quantity of viral RNA or copied DNA that was serially diluted and run at the same time as the test samples to provide a relation between cycle threshold and genome copies/mL (Appendix 1).

We determined date of symptom onset through public health, epidemiology, surveillance and laboratory records. We also calculated the number of days from symptom onset to sample collection, known as symptoms to test time, based on laboratory records (see Appendix 1).

\section{Statistical analysis}

In our previous work, ${ }^{12}$ we found that adults had a culture positivity rate of $28.9 \%$. Therefore, we required 164 pediatric samples to detect a clinically significant difference $(33 \%$ lower culture positivity rate at a power of 0.8 and $\alpha$ of 0.05 ) among children.

We present normally distributed data with means and standard deviations, and present nonnormally distributed data with medians and interquartile ranges (IQRs). We assessed normality using the Kolmorgorov-Smirnov test. We performed between-group comparisons using the Student $t$ test or the Mann-Whitney test, and used the Fisher exact test for categorical data. We compared nonparametric group medians using Kruskal-Wallis analysis of variance. We performed multivariable logistic regression using robust standard errors to test predictors of positive cultures. We considered twotailed $p$ values less than 0.05 as significant. We performed statistical analysis with Stata version 16.1 and GraphPad Prism 9.

\section{Ethics approval}

The study was performed in accordance with protocol HS23906 (H2020:211) and approved by the University of Manitoba Research Ethics Board. The ethics board waived the need for informed consent as samples were obtained as part of routine clinical and public health management and were not taken specifically for inclusion in the current study. 


\section{Results}

During the study period, about 360000 nasopharyngeal swab tests were performed in Manitoba, of which about 20000 were positive for SARS-CoV-2. Our final sample included 305 cultured specimens, representing $1.5 \%$ of positive samples in Manitoba and 7.2\% (175 of 2440) of positive samples among children. Of 175 pediatric samples cultured, 97 samples were from children 10 years old or younger and 78 were from children 11-17 years old; these were compared with 130 adult specimens. Baseline demographics, cycle thresholds and viral RNA loads are shown in Table 1 and Table 2. We successfully cultured the virus in 93 of 305 samples (31\%), including 57 of 130 adults (44\%, 95\% Cl 35\%-53\%). In comparison, we cultured the virus in only 18 of 97 samples in children 10 years old or younger $(19 \%, 95 \% \mathrm{Cl} 11 \%-28 \%, p<0.001)$ and 18 of 78 samples in children aged $11-17$ years old $(23 \%, 95 \% \mathrm{Cl} 14 \%-34 \%, p=0.003)$. The rate of positive cultures did not differ between younger and older children $(p=0.5)$. Compared with adults, children had a $55 \%$ reduced odds of growing live virus (odds ratio $0.45,95 \% \mathrm{Cl} 0.28-0.72$ ). Although children 10 years old or younger were more likely to have asymptomatic infections $(47 / 97,48 \%)$ than children $11-17$ years old $(19 / 78,24 \%)$ or adults $(9 / 130,7 \%)(p<0.001$ for all comparisons), all children aged 17 years or younger were similarly likely to be asymptomatic regardless of whether they had culture-positive or culture-negative samples (42\% v. 37\%, $p=0.9)$.

The quality of nasopharyngeal samples, as shown by the cycle threshold values of the human RNAse P gene, did not differ among the 3 age groups $(p=0.6)$. The cycle threshold of the SARS-CoV-2 E gene was lower in adults (18.7, IQR 17.9-30.4) than children 10 years old or younger (25.1, IQR 17.7-31.3, $p<0.001$ ) or children 11-17 years old (22.2, IQR 18.3-29.0, $p=0.02$ ) (Table 1 and Figure 1).

\section{Table 1: Measures of SARS-CoV-2 infectivity in children and adults}

\begin{tabular}{|c|c|c|c|c|}
\hline Variable & $\begin{array}{l}\text { Children aged } \leq 10 \mathrm{yr} \\
\qquad n=97\end{array}$ & $\begin{array}{l}\text { Children aged 11-17 yr } \\
\qquad n=78\end{array}$ & $\begin{array}{l}\text { Adults } \\
n=130\end{array}$ & $p$ value \\
\hline Asymptomatic, no. (\%) & $47(48)$ & $19(24)$ & $9(7)$ & $<0.001 \S$ \\
\hline Positive culture, no. $(\%, 95 \% \mathrm{Cl})$ & $18(19,11-28)$ & $18(23,14-34)$ & $57(44,35-53)$ & $<0.0019$ \\
\hline Symptom to test time, median (IQR), d & $1(1-4)$ & $2(1-3.5)$ & $2(1-4)$ & 0.6 \\
\hline Cycle threshold ${ }^{\star}$, median (IQR) & $25.1(17.7-31.3)$ & $22.2(18.3-29.0)$ & $18.7(17.9-30.4)$ & $<0.001^{\star \star}$ \\
\hline RNAseP†, mean \pm SD & $25.7 \pm 2.8$ & $26.1 \pm 2.6$ & $26.1 \pm 2.0$ & 0.6 \\
\hline $\mathrm{TCID}_{50} / \mathrm{mL} \ddagger$, median (IQR) & $1171(316-5620)$ & $316(178-2125)$ & $5620(1171-17800)$ & $<0.001 \dagger \dagger$ \\
\hline Log RNA copies/mL, median (IQR) & $5.4(3.5-7.8)$ & $6.4(4.2-7.6)$ & $7.5(5.2-8.3)$ & $<0.001 \ddagger \ddagger$ \\
\hline \multicolumn{5}{|c|}{ 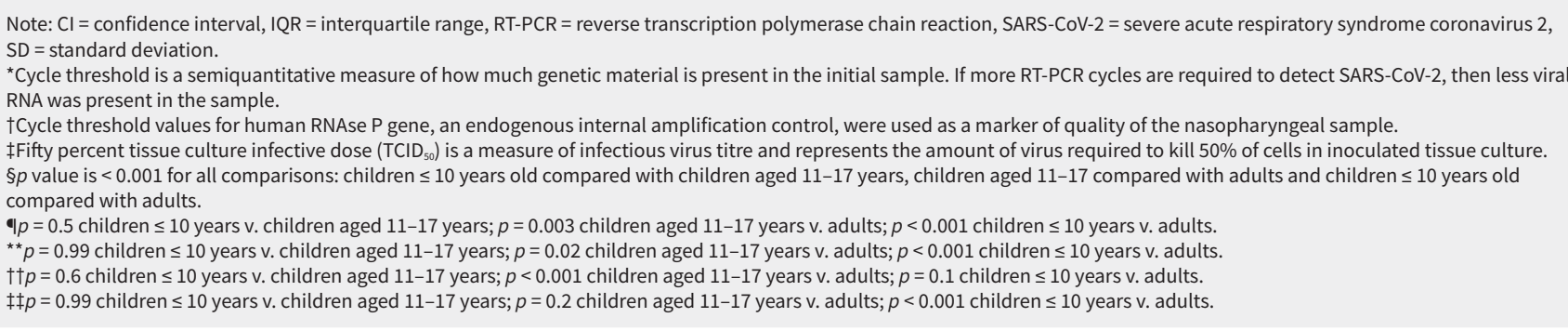 } \\
\hline
\end{tabular}

\section{Table 2: Measures of SARS-CoV-2 infectivity in pediatric culture-positive versus culture-negative samples}

\begin{tabular}{|c|c|c|c|}
\hline Variable & $\begin{array}{l}\text { No. (\%) of culture-positive samples* } \\
\qquad n=36\end{array}$ & $\begin{array}{l}\text { No. (\%) of culture-negative samples* } \\
\qquad n=139\end{array}$ & $p$ value \\
\hline Age, yr, median (IQR) & $10(5-15)$ & $9(5-14)$ & 0.6 \\
\hline Asymptomatic & $15(42)$ & $51(37)$ & 0.9 \\
\hline Male sex & $22(61)$ & $80(57)$ & 0.7 \\
\hline Symptom to test time, median (IQR), d & $1(0-2)$ & $2(1-4)$ & 0.3 \\
\hline Cycle thresholdt, median (IQR) & $16.8(16.3-18.8)$ & $25.8(20.7-31.9)$ & $<0.001$ \\
\hline Log RNA copies/mL, median (IQR) & $8.1(7.4-8.2)$ & $5.2(3.2-6.8)$ & $<0.001$ \\
\hline
\end{tabular}




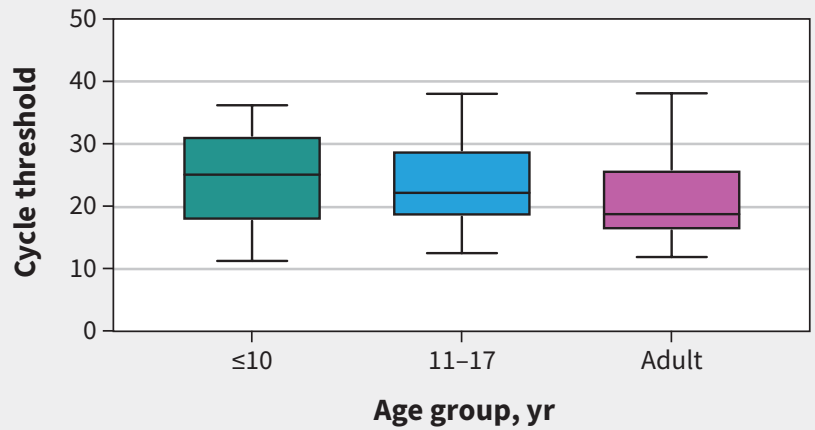

Figure 1: Reverse transcription polymerase chain reaction cycle threshold values of the severe acute respiratory syndrome coronavirus 2 envelope gene by age group. Adult samples had a significantly lower cycle threshold value (18.7, interquartile range [IQR] 17.9-30.4) than children aged $\leq 10$ years $(25.1$, IQR $17.7-31.3, p<0.001)$ and those aged $11-17$ years (22.2, IQR 18.3-29.0, $p=0.02)$.

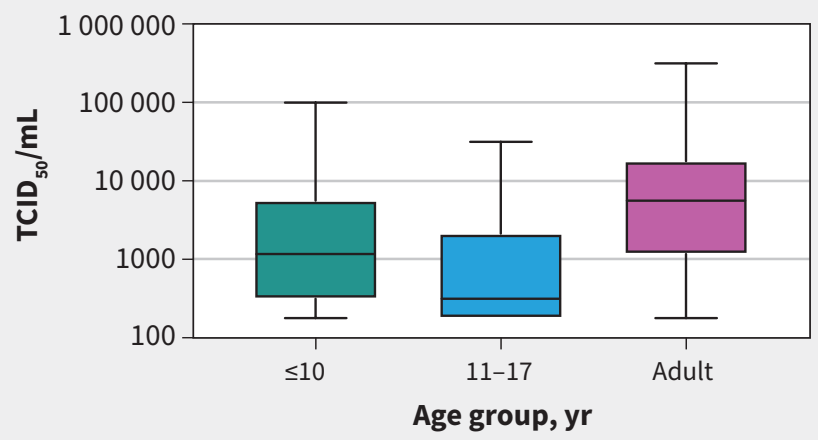

Figure 2: Tissue culture infective dose $50 \%\left(\mathrm{TCID}_{50} / \mathrm{mL}\right)$ by age group. Adult samples had significantly higher $\mathrm{TCID}_{50} / \mathrm{mL}$ (5620, IQR 1171-17800) than children aged 11-17 years (316, interquartile range [IQR] 178-2125, $p<0.001$ ), but were not significantly higher than children aged $\leq 10$ years (1171, IQR 316 to $5620, p=0.1$ ).

The median $\mathrm{TCID}_{50} / \mathrm{mL}$ was significantly lower for children aged 11-17 years (316, IQR 178-2125) than adults (5620, IQR $1171-17800, p<0.001)$, but differences between adults and children 10 years old or younger (1171, IQR 316-5620, $p=0.1$ ) did not reach statistical significance (Table 1 and Figure 2 ).

There was no difference between pediatric culture-positive and culture-negative samples, except for cycle threshold values and log RNA copies/mL (Table 2). The median cycle threshold was lower in culture-positive samples (16.8, IQR 16.3-18.8) than culture-negative samples (25.8, IQR 20.7-31.9, $p<0.001)$. The median log RNA copies/mL was higher in culture-positive samples 8.1, IQR 7.4-8.2) than culture-negative samples (5.2, IQR $3.2-6.8, p<0.001)$. However, the median symptoms to test time was not different between the culture-positive (1 d, IQR 0-2 d) and culture-negative groups ( $2 \mathrm{~d}$, IQR $1-4 \mathrm{~d}, p=0.3$ ). The probability of a positive culture varied by symptoms to test time, with likelihood of a positive culture being highest among speci-

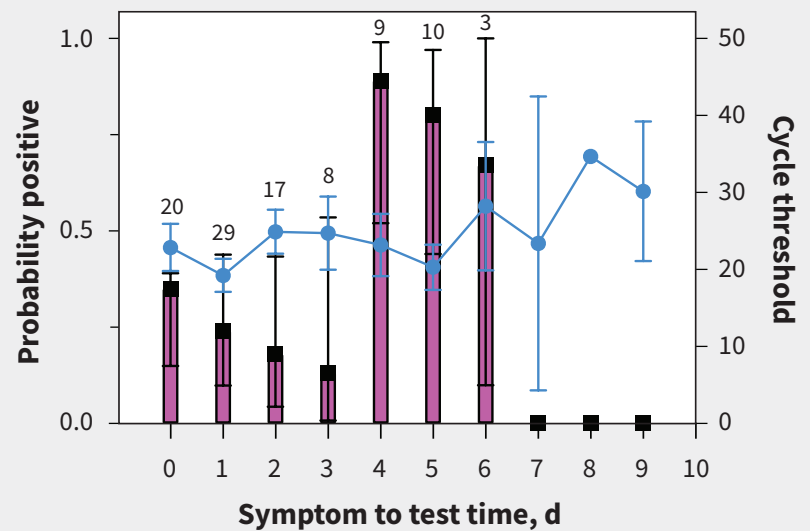

Figure 3: Symptom onset to test time (days), the mean revere transcription polymerase chain reaction cycle threshold value of the severe acute respiratory syndrome coronavirus 2 (SARS-CoV-2) envelope gene and the probability of successful viral culture in pediatric samples. The probability of SARS-CoV-2 culture is shown by the pink bars. Black lines represent $95 \%$ confidence intervals. Cycle threshold values are represented by the blue line, with circles representing medians and blue bars representing the $95 \%$ confidence intervals. Numbers above the pink bar indicate the number of samples per day.

mens collected 4-6 days after symptom onset, whereas cycle threshold showed less variation across symptoms to test times (Figure 3).

Receiver operating characteristic (ROC) analysis of the cycle threshold to discriminate between children with and without positive viral culture showed an area under the receiveroperator curve (AUC) of 0.87 (95\% Cl 0.81-0.93) (Appendix 1, Supplementary Figure 1). The specificity of a cycle threshold of 23 was $97.2 \%$ (95\% Cl 85.8\%-99.9\%) (Appendix 1, Supplementary Table 1). Similar results were seen in adults (AUC 0.89, 95\% $\mathrm{Cl} 0.83-0.96, p=0.6 \mathrm{v}$. children) (Appendix 1, Supplmentary Figure 1, Supplementary Table 2). Symptoms to test time was not as accurate as cycle threshold in discriminating between samples with and without positive viral culture (children, AUC $0.67,95 \% \mathrm{Cl} 0.55$ to $0.79 \mathrm{v}$. adults, AUC $0.78,95 \% \mathrm{Cl} 0.68-0.88$, $p=0.2$ ) (Appendix 1, Supplementary Figure 2), with a specificity of $100 \%(95 \% \mathrm{Cl} 84.5 \%-100 \%)$ at a symptoms to test time of greater than 6 days. Of note, our sample had only 8 patients with symptoms to test time of 6 days or more, calling into question the accuracy of the results in determining a cut-off period of symptoms to test time for cell culture positivity and possible infectivity because of a lack of power.

Multivariable logistic regression showed that, for pediatric samples, cycle threshold was an independent predictor of positive culture (odds ratio $0.81,95 \% \mathrm{Cl} 0.69-0.94$ ), but symptoms to test time, age and sex were not (Table 3).

In a supplementary analysis, we found no difference in the culture-positive rates between children aged 0-4 years compared with children aged 5-10 years. The level of virus (based on $\mathrm{TCID}_{50} / \mathrm{mL}$ ) also did not differ among culture-positive samples from children in these 2 age groups. 
Table 3: Multivariable logistic regression model of measures associated with a positive viral culture from pediatric samples
Cycle threshold*

Symptoms to test time

Age

Sex

RNAsePt
Variable
Adjusted odds ratio $(95 \% \mathrm{Cl})$

$0.81(0.69-0.94)$

$0.90(0.64-1.27)$

$1.13(0.97-1.31)$

$2.18(0.48-9.87)$

$0.69(0.48-1.00)$
Note: $\mathrm{Cl}$ = confidence interval, $\mathrm{RT}-\mathrm{PCR}=$ reverse transcription polymerase chain reaction, SARS-CoV-2 = severe acute respiratory syndrome coronavirus 2 .

${ }^{*}$ Cycle threshold is a semiquantitative measure of how much genetic material is present in the initial sample. If more RT-PCR cycles are required to detect SARS-CoV-2, then less viral RNA was present in the sample.

†Cycle threshold values for human RNAse P gene, an endogenous internal

amplification control, were used as a marker of quality of the nasopharyngeal sample.

\section{Interpretation}

Our results show that samples from 175 children 17 years old or younger had about half the odds of containing culturable virus than samples from adults. When SARS-CoV-2 was successfully cultured, the median $\mathrm{TCID}_{50} / \mathrm{mL}$ was significantly lower for pediatric samples than adults, meaning that less viable virus was present. Moreover, the culture positivity rate of samples from children 10 years or younger was significantly lower than for children aged 11-17 years or adults. These results illustrate that RT-PCR positivity does not necessarily equate to culture positivity, as RT-PCR positivity alone does not distinguish between live virus in an infectious patient and residual viral RNA in a patient who may no longer be infectious.

We found that the cycle threshold value was highly predictive of culture positivity. In contrast, symptoms to test time was not able to discriminate between children with positive and negative cultures. Thus, in children who have tested positive for SARS-CoV-2 by RT-PCR, knowing the cycle threshold value may be more informative for determining the potential infectiousness of a child, and may have implications for duration of isolation.

These results are contrary to what has been observed with other respiratory viruses for which efficient infection and transmission in children often herald widespread community transmission. However, these findings are consistent with epidemiological studies that show limited SARS-CoV-2 spread from children younger than 10 years old. ${ }^{16,17}$ A recent seroprevalence study from Germany showed that children, particularly those aged 1-10 years, have a significantly lower seropositivity than their parents, making undetected asymptomatic infections in children less likely. ${ }^{18} \mathrm{~A}$ meta-analysis showed that children have a lower susceptibility to SARS-CoV-2 and may not drive community transmission to the same degree as adults. ${ }^{19}$ Severe acute respiratory syndrome coronavirus 2 also has an overly dispersed reproduction number $\left(\mathrm{R}_{0}\right)$, suggesting that its transmission dynamics are fundamentally different than epidemic seasonal respiratory viruses..$^{20-22}$ Overdispersion refers to high individual-level variation in the distribution of the number of secondary transmissions, which can lead to so-called "superspreading" events. ${ }^{21}$

Others have looked at the ability to grow live virus from pediatric samples, the gold standard for microbiological diagnosis. L'Huillier and colleagues grew live virus from a higher proportion of pediatric samples than our study (52\%; $12 / 23$, v. $31 \%$ in our combined samples) ${ }^{11}$ Their findings of symptoms to test time were similar to what we observed, and the $95 \%$ Cls of our results almost overlap theirs, suggesting that their smaller sample size may be responsible for their higher proportion of culture-positive results. However, closer inspection of the L'Huillier data reveals that, consistent with our current study, culture positivity varied with age, such that virus was cultured from only 4 of 11 (36.4\%) children 10 years old or younger, but 8 of $12(66.6 \%)$ children 11 years old or older. Recognizing that cycle threshold is a limited surrogate of viral load, other studies have attempted to further quantify viral load in children by using log RNA copies/mL based on standardized curves. Although this approach does improve the ability to compare data across time and laboratories, it remains a surrogate measure of viable viral load, cannot predict recoverable live virus and is vulnerable to being confounded by shedding of noninfectious viral genetic material. As such, inferences from measures of viral load derived from cycle threshold data have substantial limitations. We quantified viral presence through the use of $\mathrm{TCID}_{50} / \mathrm{mL}$, which provides additional discriminatory power compared with methods that limit the analysis to simply the presence or absence of cytopathic effect. ${ }^{11}$

The observation of a cycle threshold value greater than 23 signalling a significant reduced risk of recovering live virus is worth examining in a larger study. This value is in keeping with our previous work that showed a decreased ability to grow live virus in adult samples where the cycle threshold was greater than $24 .{ }^{12}$ Finally, defining a robust symptom-based cut-off period for cell culture positivity should be undertaken, although testing often occurs shortly after symptom onset. It may prove challenging to answer this question in the current COVID-19 testing environment.

\section{Limitations}

Other possible explanations for our findings should be considered. Viral genetic variation may play a role; however, genomic surveillance shows that samples represented the diverse global lineages present in the initial and subsequent waves of cases in Manitoba. Sample collection from children can be challenging, resulting in a suboptimal specimen. The lack of significant differences in cycle threshold values of RNAse P (an endogenous internal control), however, suggests similar sampling quality across age groups. Degradation of samples while in storage, affecting the chance of viral recovery, was also considered, but the time to cell culture was similar across age groups, making this possibility unlikely.

Although young children had similar symptoms to test time, children may be most infectious at a different time postexposure than adolescents or adults. Our local epidemiology (unpublished data, 2021) does not support this argument, as pediatric cases of COVID19 are consistent with community transmission. ${ }^{23,24}$ It is possible that children were at a different point in their viral trajectory relative to adolescents and adults when they were sampled. As only a single sample was taken, it would not be possible to determine the longitudinal trend in cycle threshold value relative to sampling time. Regression analysis (data not shown) from adults and children did not show any correlation between symptoms to test time and cycle threshold or $\mathrm{TCID}_{50} / \mathrm{mL}$ value. Recall bias of symptom onset is possible and symptoms may be subtle in children, thus compounding 
recall bias, but this is likely equally distributed among all patients. Finally, we cannot be certain that our findings apply to novel SARSCoV-2 variants that have shown higher levels of infectivity, as such variants were not commonly circulating during the study period.

\section{Conclusion}

We found that SARS-CoV-2 grew from pediatric samples less often than adult samples, and when the virus was successfully cultured, significantly less viable virus was present. These data, along with our local epidemiology, suggest that children do not appear to be the main drivers of SARS-CoV-2 transmission. Our findings have important public health and clinical implications. If younger children are less capable of transmitting infectious virus, daycare, in-person school and cautious extracurricular activities may be safe to continue, with appropriate precautions in place, and with lower risk to child care staff, educators and support staff than initially anticipated. Given the difficulties in keeping children isolated within the home environment and the significant impact of prolonged isolation on both child development and parental function (such as loss of work or income), a robust tool to decrease the length of, or need for, quarantine would be an important public health development.

\section{References}

1. Reichert TA, Sugaya N, Fedson DS, et al. The Japanese experience with vaccinating schoolchildren against influenza. N Engl J Med 2001;344:889-96.

2. Baggio S, L'Huillier AG, Yerly S, et al. SARS-CoV-2 viral load in the upper respiratory tract of children and adults with early acute COVID-19. Clin Infect Dis 2020 Aug. 6; ciaa1157. doi: 10.1093/cid/ciaa1157. [Epub ahead of print].

3. Guilamo-Ramos V, Benzekri A, Thimm-Kaiser M, et al. Reconsidering assumptions of adolescent and young adult SARS-CoV-2 transmission dynamics. Clin Infect Dis 2020; Sept. 7;ciaa1348. doi: 10.1093/cid/ciaa1348. [Epub ahead of print].

4. Lopez AS, Hill M, Antezano J, et al. Transmission dynamics of COVID-19 outbreaks associated with child care facilities - Salt Lake City, Utah, April-July 2020. MMWR Morb Mortal Wkly Rep 2020;69:1319-23.

5. Schmidt E, Steinhagen K, Rupp J. Heavy exposure of children aged 9-12 years with severe acute respiratory syndrome coronavirus 2 did not lead to infection. J Pediatric Infect Dis Soc 2020;9:620-1.

6. Stein-Zamir C, Abramson N, Shoob H, et al. A large COVID-19 outbreak in a high school 10 days after schools' reopening, Israel, May 2020. Euro Surveill 2020;25:2001352.
7. Szablewski CM, Chang KT, Brown MM, et al. SARS-CoV-2 transmission and infection among attendees of an overnight camp - Georgia, June 2020. MMWR Morb Mortal Wkly Rep 2020;69:1023-5.

8. Yonker LM, Neilan AM, Bartsch Y, et al. Pediatric severe acute respiratory syndrome coronavirus 2 (SARS-CoV-2): clinical presentation, infectivity, and immune responses. J Pediatr 2020;227:45-52 e5.

9. Zhang J, Litvinova M, Liang $\mathrm{Y}$, et al. Changes in contact patterns shape the dynamics of the COVID-19 outbreak in China. Science 2020;368:1481-6.

10. Hurst JH, Heston SM, Chambers HN, et al. SARS-CoV-2 infections among children in the Biospecimens from Respiratory Virus-Exposed Kids (BRAVE Kids) study. Clin Infect Dis 2020; Nov. 3; ciaa1693. doi: 10.1093/cid/ciaa1693. [Epub ahead of print].

11. L'Huillier AG, Torriani G, Pigny F, et al. Culture-competent SARS-CoV-2 in nasopharynx of symptomatic neonates, children, and adolescents. Emerg Infect Dis 2020;26:2494-7.

12. Bullard J, Dust K, Funk D, et al. Predicting infectious SARS-CoV-2 from diagnostic samples. Clin Infect Dis 2020;71:2663-6.

13. Wolfel R, Corman VM, Guggemos W, et al. Virological assessment of hospitalized patients with COVID-2019. Nature 2020;581:465-9.

14. DeBiasi RL, Delaney M. Symptomatic and asymptomatic viral shedding in pediatric patients infected with severe acute respiratory syndrome coronavirus 2 (SARS-CoV-2): under the surface. JAMA Pediatr 2021;175:16-8.

15. Fantini MP, Reno C, Biserni GB, et al. COVID-19 and the re-opening of schools: a policy maker's dilemma. Ital J Pediatr 2020;46:79.

16. Heavey L, Casey G, Kelly C, et al. No evidence of secondary transmission of COVID19 from children attending school in Ireland, 2020. Euro Surveill 2020;25:2000903.

17. Zimmermann P, Curtis N. Coronavirus infections in children including COVID19: an overview of the epidemiology, clinical features, diagnosis, treatment and prevention options in children. Pediatr Infect Dis J 2020;39:355-68.

18. Tönshoff B, Müller B, Elling R, et al. Prevalence of SARS-CoV-2 infection in children and their parents in Southwest Germany. JAMA Pediatr 2021 Jan. 22; e210001. doi: 10.1001/jamapediatrics.2021.0001. [Epub ahead of print].

19. Viner RM, Mytton OT, Bonell C, et al. Susceptibility to SARS-CoV-2 infection among children and adolescents compared with adults: a systematic review and meta-analysis. JAMA Pediatr 2021;175:143-56.

20. Lau MSY, Grenfell B, Thomas M, et al. Characterizing superspreading events and age-specific infectiousness of SARS-CoV-2 transmission in Georgia, USA. Proc Natl Acad Sci U S A 2020;117:22430-5.

21. Lloyd-Smith JO, Schreiber SJ, Kopp PE, et al. Superspreading and the effect of individual variation on disease emergence. Nature 2005;438:355-9.

22. Adam DC, Wu P, Wong JY, et al. Clustering and superspreading potential of SARS-CoV-2 infections in Hong Kong. Nat Med 2020;26:1714-9.

23. Information for Manitobans: COVID-19 dashboard: school-aged cases and school staff cases in Manitoba. Winnipeg: Government of Manitoba. Available: https://www.gov.mb.ca/covid19/schools/index.html (accessed 2021 Feb. 8).

24. Reports and updates: weekly COVID-19 and seasonal influenza report. Winnipeg: Government of Manitoba. Available: https://www.gov.mb.ca/health/ publichealth/surveillance/reports.html (accessed 2021 Feb. 8).
Competing interests: Lauren Garnett, Kaylie Tran, Alex Bello and James Strong report A-base funding from the Government of Canada. No other competing interests were declared.

This article has been peer reviewed.

Affiliations: Cadham Provincial Laboratory (Bullard, Dust, Hedley, Alexander, Van Caeseele), Manitoba Health; Department of Pediatrics \& Child Health (Bullard, Strong, Lee, Van Caeseele, Poliquin), University of Manitoba; National Microbiology Laboratory (Funk, Garnett, Tran, Bello, Strong, Poliquin), Public Health Agency of Canada; Departments of Anesthesiology and Medicine (Funk), Section of Critical Care, University of Manitoba; Department of Medical Microbiology \& Infectious Diseases (Garnett, Tran, Bello, Alexander), University of Manitoba; Communicable Disease Control, Public Health (Lee), Manitoba Health; Epidemiology and Surveillance Unit (Waruk, Loeppky), Manitoba Health; Department of Community Health Science (Loeppky), University of Manitoba. Winnipeg, Man.

Contributors: All of the authors contributed to the conception and design of the work, and the acquisition, analysis, and interpretation of data. All of the authors drafted the manuscript, revised it critically for important intellectual content, gave final approval of the version to be published and agreed to be accountable for all aspects of the work.
Content licence: This is an Open Access article distributed in accordance with the terms of the Creative Commons Attribution (CC BY-NC-ND 4.0) licence, which permits use, distribution and reproduction in any medium, provided that the original publication is properly cited, the use is noncommercial (i.e., research or educational use), and no modifications or adaptations are made. See: https://creativecommons.org/licenses/by-nc-nd/4.0/

Data sharing: Data with potential personal health information cannot be shared by Manitoba Health and are regulated by the Health Information Privacy Committee (HIPC) under the Public Health Information Act of Manitoba. Upon request, data that have been appropriately anonymized and deidentified may be provided to researchers through consultation with the corresponding author.

Acknowledgments: This work was supported by the collaborative efforts in the public health response to the COVID-19 pandemic by Manitoba Health, Cadham Provincial Laboratory, the Public Health Agency of Canada and the National Microbiology Laboratory. The authors also thank the teachers and educators, who have maintained some normalcy for the children of Manitoba, and the children themselves.

Accepted: Mar. 30, 2021

Correspondence to: Jared Bullard, jared.bullard@gov.mb.ca 